VOL. 47 (1993) [377-384]

\title{
SOME CLASSES OF SEQUENTIAL SPACES
}

\author{
Sergey A. Svetlichny
}

\begin{abstract}
By means of defined concepts all metric spaces of given weight or cardinality and their quotients are characterised. An example of a sequential space having weight $\omega_{1}$ which is not a quotient of any metric space of weight $\omega_{1}$ is provided. The wellknown classes of sequential spaces are also obtained as images of metric spaces by particular kinds of maps.
\end{abstract}

\section{INTRODUCTION}

The purpose of this note is to give some new approaches to the theory of sequential spaces. We shall introduce and consider the concept of a sequential base which may deserve to be better known.

Originally, the results of this paper were stimulated by the following question of Arhangel'skii that was formulated at Moscow Seminars on Topology:

QUESTION 1.1. Is every sequential space $X$ of weight $\tau$ a quotient of some metric space $M$ of weight $\tau$ ?

It is easy to show that, whenever $\tau^{\omega}=\tau$, the disjoint union of $\tau$ copies of the converging sequence will serve as a metrisable space $M$ of weight $\tau$ for a space $X$ of weight $\tau$. However, as we show below, Question 1.1 cannot be answered affirmatively using no axioms beyond ZFC.

It may also be of interest to point out that not every countable sequential space is a quotient of some countable metric space. This follows, for instance, from the following result:

EXAMPLE 1.2: [7] There exists a countable sequential space which is not a quotient of any metric space of cardinality less than $2^{\omega}$.

In this paper we shall use the terminology and notation from [2]. As usual, an ordinal is equal to the set of all smaller ordinals, $\omega$ is $\omega_{0}$ and a cardinal is an initial ordinal. We use MA and CH to denote Martin's axiom and the continuum hypothesis respectively. All spaces are assumed to be regular, hence $T_{1}$, all maps are continuous and onto.

Received 30 April 1992

The author wants to express his deep gratitude to Professor A.V. Arhangel'skii for helpful discussions.

Copyright Clearance Centre, Inc. Serial-fee code: 0004-9729/93 \$A2.00+0.00. 


\section{METRIC SPACES OF GIVEN WEIGHT OR CARDINALITY}

\section{AND THEIR QUOTIENTS}

First let us define the following main concept.

Definition 2.1: For every topological space $X$, a collection $\mathcal{P}$ of subsets of $X$ is called a sequential base if for every point $x \in X$ one can assign a collection $\mathcal{P}_{x} \subseteq \mathcal{P}^{\omega}$ such that:

(i) if $\left(P_{n}\right) \in \mathcal{P}_{x}$, then $x \in \bigcap_{n} P_{n}$ and $P_{n+1} \subseteq P_{n}$ for each $n \in \omega$,

(ii) a set $V$ is open in $X$ if and only if for every point $x \in V$ and every $\left(P_{n}\right) \in \mathcal{P}_{x}$ there exists $m \in \omega$ such that $P_{m} \subseteq V$.

Proposition 2.2. A space $X$ is sequential if and only if $X$ has a sequential base.

ProOf: The collection of all converging sequences (including their limits) of a sequential space $X$ provides the sequential base for $X$. The converse implication is evident.

THEOREM 2.3. The following are equivalent for a space $X$ and any cardinal $\tau$ :

(a) $X$ is a quotient of a metric space having weight $\tau$.

(b) $X$ has a sequential base of cardinality $\tau$.

Proof: $(\mathrm{a}) \Rightarrow(\mathrm{b})$. Let $\mathcal{B}$ be a base of cardinality $\tau$ for the metric space $M$ and let $f: M \rightarrow X$ be a quotient map. For each $x \in X$ and $\xi \in f^{-1}(x)$, let $\left\{B_{n}(\xi): n \in\right.$ $\omega\} \subseteq \mathcal{B}$ be a countable base at $\xi \in M$ and denote $P_{n}(\xi)=f\left(B_{n}(\xi)\right)$ for all $n \in \omega$. It is easy to check that $\mathcal{P}=\{f(B): B \in \mathcal{B}\}$ satisfies the conditions of Definition 2.1 if the collection $\mathcal{P}_{z}=\left\{\left(P_{n}(\xi)\right) \in \mathcal{P}^{\omega}: \xi \in f^{-1}(x)\right\}$ is assigned for each $x \in X$. So $X$ has the sequential base $\mathcal{P}$ of cardinality $\leqslant \tau$. Then $X$ has some sequential base of cardinality $\tau$.

(b) $\Rightarrow$ (a). Suppose $X$ has a sequential base $\mathcal{P}$ of cardinality $\tau \geqslant \omega$ (the case $\tau<\omega$ is evident) and endow $\mathcal{P}$ with the discrete topology. Then $\mathcal{P}^{\omega}$ is a metrisable space of weight $\tau$. Now for every $x \in X$, let $\mathcal{P}_{x}^{*}$ be the set of all $\left(P_{n}^{*}\right) \in \mathcal{P}^{w}$ such that $x \in \bigcap_{n} P_{n}^{*}$ for all $n \in \omega$ and there is $\left(P_{n}\right) \in \mathcal{P}_{x}$ with $P_{n}^{*}=P_{n}$ for all but finitely many $n \in \omega$. Let $M=\bigcup\left\{\mathcal{P}_{x}^{*}: x \in X\right\} \subseteq \mathcal{P}^{\omega}$ and define the map $f: M \rightarrow X$ by the rule: $f\left(\left(P_{n}^{*}\right)\right)=x$ if $\left(P_{n}^{*}\right) \in \mathcal{P}_{x}^{*}$. Then, evidently, our map $f: M \rightarrow X$ is onto. To prove that $f$ is continuous let $U \subseteq X$ be an open set, $\left(P_{n}^{*}\right) \in M$ and $f\left(\left(P_{n}^{*}\right)\right) \in U$. Pick $m \in \omega$ such that $P_{m}^{*} \subseteq U$. Consider the following open set $\prod_{n} W_{n} \subseteq \mathcal{P}^{\omega}: W_{n}=\mathcal{P}$ if $n \neq m$, and $W_{m}=\left\{P_{m}^{*}\right\}$. Then $f\left(M \cap \prod_{n} W_{n}\right)=P_{m}^{*} \subseteq U$. So $\left(M \cap \prod_{n} W_{n}\right) \subseteq f^{-1}(U)$ and $f^{-1}(U)$ is open in $M$. 
It remains to show that $f$ is quotient. Suppose $U \subseteq X$ and $f^{-1}(U)$ is open in $M$. Consider any $x \in X$ and $\left(P_{n}\right) \in \mathcal{P}_{z}$. Since $\left(P_{n}\right) \in f^{-1}(U)$, find $\left\{n_{i}: i<k\right\} \subseteq \omega$ and $\prod_{n} W_{n} \subseteq \mathcal{P}^{\omega}$ such that $W_{n}=\left\{P_{n}\right\}$ if $n \in\left\{n_{i}: i<k\right\}, W_{n}=\mathcal{P}$ otherwise and $\left(M \cap \prod_{N} W_{n}\right) \subseteq f^{-1}(U)$. Let $m=\max \left\{n_{i}: i<k\right\}$. Then $P_{m}=f\left(M \cap \prod_{n} W_{n}\right) \subseteq$ $U$. So $U$ is open in $X$.

We have proved that $X$ is a quotient of the metrisable space $M$ having weight $\leq \tau$. So $X$ is a quotient of the disjoint union of $\tau$ copies of $M$, which is a metrisable space having weight $\tau$.

Corollary 2.4. A space $X$ is a sequential $\aleph_{0}$-space [2] if and only if $X$ has a countable sequential base.

COROLlary 2.5. Each of the following two conditions implies that $X$ is a quotient of a metric space having weight $\tau$ :

(a) $X$ is a first-countable space having weight $\tau$.

(b) $X$ is the one-point compactification of an uncountalbe discrete space of cardinality $\tau$.

Proof: (of (b).) Let $X=\{\alpha: \alpha \leq \tau\}$, where the point $\tau$ is non-isolated. For any limit $\beta<\tau, \operatorname{cof}(\beta)=\omega$ choose a sequence $\left(\alpha_{n}^{\beta}\right)$ converging to $\beta$ in the usual order topology and define

$$
\begin{aligned}
P_{n}^{\beta} & =\{\tau\} \cup\left\{\lambda: \alpha_{n}^{\beta}<\lambda<\beta\right\}, \\
\mathcal{P} & =\{\{\alpha\}: \alpha<\tau\} \cup\left\{P_{n}^{\beta}: n \in \omega, \beta<\tau, \operatorname{cof}(\beta)=\omega\right\} .
\end{aligned}
$$

For every $\alpha \in X$, let $\mathcal{P}_{\alpha}=\left\{\left(\alpha_{n}\right): \alpha_{n}=\alpha\right\}$ if $\alpha<\tau$ and $\mathcal{P}_{\alpha}=\left\{\left(P_{n}^{\beta}\right): \beta<\right.$ $\tau, \operatorname{cof}(\beta)=\omega\}$ if $\alpha=\tau$. It is a routine verification to show that $|\mathcal{P}|=\tau$ and $\mathcal{P}$ is a sequential base of $X$ if $\mathcal{P}_{\alpha}$ is considered to be the assigned collection for $\alpha \leq \tau$ as in Definition 2.1.

Now we shall consider quotients of metric spaces of given cardinalities. In [7] a countable sequential space which is not a quotient of any metric space of cardinality less than $2^{\omega}$ was exhibited.

Definition 2.6: Let $\mathcal{P}$ be a sequential base of a space $X$. Then the fan-number of $\mathcal{P}$, denoted $f n(\mathcal{P})$, is the smallest cardinal $\tau$ such that for each $x \in X$, the collection $\mathcal{P}_{x}$ from Definition 2.1 may be chosen of cardinaltiy $\leq \tau$.

REMARK. If $X$ has a sequential base with finite fan-number then $X$ has also a sequential base $\mathcal{P}$ such that $f n(\mathcal{P})=1$.

THEOREM 2.7. The following are equivalent for a space $X$ and any cardinal $\tau$ :

(a) $X$ is a quotient of a metric space having cardinality $\tau$. 
(b) $|X| \leqslant \tau$ and $X$ has a sequential base $\mathcal{P}$ such that $f n(\mathcal{P}) \leqslant \tau$.

ProOF: (a) $\Rightarrow(b)$. As in the proof of (a) $\Rightarrow(b)$ in Theorem 2.3, we consider the quotient map $f: M \rightarrow X$ and the sequential base $\mathcal{P}=\{f(B): B \in \mathcal{B}\}$. Since $\left|f^{-1}(x)\right| \leqslant \tau$ for every $x \in X$, it follows that $f n(\mathcal{P}) \leqslant \tau$.

(b) $\Rightarrow$ (a). Suppose $X$ has a sequential base $\mathcal{P}$ such that $f n(\mathcal{P}) \leqslant \tau$. Since $|X| \leqslant \tau$ and $f n(\mathcal{P}) \leqslant \tau$, it follows that $|\mathcal{P}| \leqslant \tau$. By the construction from (b) $\Rightarrow$ (a) in Theorem 2.3, we have the metric space $M=\bigcup\left\{\mathcal{P}_{x}^{*}: x \in X\right\}$ and the quotient map $f: M \rightarrow X$. From $|\mathcal{P}| \leqslant \tau$ it follows that $\left|\mathcal{P}_{x}^{*}\right| \leqslant \tau$ for each $x \in X$, so $|M| \leqslant \tau$. Hence $X$ is a quotient of the disjoint union of $\tau$ copies of $M$, which is a metrisable space having cardinality $\tau$.

Using Definition 2.6 we say that a space $X$ is $g$-first countable $[1,6]$ if $X$ has a sequential base $\mathcal{P}$ with finite fan-number, or $f n(\mathcal{P})=1$ (see Remark), and a space $X$ is weakly-quasi-first-countable [6] if $X$ has a sequential base $\mathcal{P}$ with countable fannumber, or $f n(\mathcal{P})=\omega$.

Corollary 2.8. If $X$ is a $g$-first countable space of cardinality $\tau$ then $X$ is a quotient of metric space of cardinality $\tau$.

Corollary 2.9. [6] A countable space $X$ is a quotient of a countable metric space if and only if it is weakly-quasi-first-countable.

\section{AN EXAMPLE}

The purpose of this section is to construct an example which answers Question 1.1 in the negative. We first record the necessary definitions.

As usual, $\omega_{\omega} \omega$ denotes the set of all functions from the natural numbers $\omega$ into the natural numbers. For any $f, g \in \omega^{\omega} \omega$, we write $f<^{*} g$ if $f(n)<g(n)$ for all but finitely many $n \in \boldsymbol{\omega}$.

Definition 3.1: A system $\left\{f_{\alpha}: \alpha<\tau\right\} \subseteq \omega^{\omega} \omega$ is said to be increasing if $f_{\lambda}<f_{\beta}$ for all $\lambda<\beta<\tau$.

Definition 3.2: A function $g \in \omega_{\omega} \omega$ is called a majorant of a system $\left\{f_{\alpha}: \alpha<\right.$ $\tau\} \subseteq{ }^{\omega} \omega$ if $f_{\alpha}<^{*} g$ whenever $\alpha<\tau$.

LEMma 3.3. (MA $+\neg C H)$ Suppose each function of the system $\left\{g_{\beta}: \beta<\omega_{1}\right\} \subseteq$ $\omega_{\omega}$ is a majorant of an increasing system $\left\{f_{\alpha}: \alpha<\omega_{1}\right\} \subseteq \omega_{\omega}$. Then there are $\varphi \in{ }^{\omega} \omega$ and an infinite $\left\{n_{k}: k \in \omega\right\} \subseteq \omega$ which satisfy the condition:

(i) for any $\alpha, \beta<\omega_{1}$ there is $k(\alpha, \beta) \in \omega$ such that $f\left(n_{k}\right)<\varphi\left(n_{k}\right)<g\left(n_{k}\right)$ for all $k>k(\alpha, \beta)$. 
PROOF: Let

$$
\begin{aligned}
A_{\alpha} & =\left\{\langle i, j\rangle \in \omega \times \omega: j>f_{\alpha}(i)\right\}, \\
B_{\beta} & =\left\{\langle i, j\rangle \in \omega \times \omega: j<g_{\beta}(i)\right\}, \\
\boldsymbol{H}_{\alpha, \beta} & =\left(A_{\alpha} \cap B_{\beta}\right) \subseteq \omega \times \omega .
\end{aligned}
$$

Since $g_{\beta}$ is a majorant of $\left\{f_{\alpha}: \alpha<\omega_{1}\right\}$ for any $\beta<\omega_{1}$ and $\left\{f_{\alpha}: \alpha<\omega_{1}\right\}$ is increasing, we have that $H_{\alpha, \beta}$ is infinite whenever $\alpha, \beta<\omega_{1}$.

Now consider the collection $\mathcal{H}=\left\{H_{\alpha, \beta} \subseteq \omega \times \omega: \alpha, \beta<\omega_{1}\right\}$ and any $H_{\alpha_{1}, \beta_{1}} \in \mathcal{H}$, $H_{\alpha_{2}, \beta_{2}} \in \mathcal{H}$. Let $\alpha=\max \left\{\alpha_{1}, \alpha_{2}\right\}$ and $g(n)=\min \left\{g_{\beta_{1}}(n), g_{\beta_{2}}(n)\right\}$ for all $n \in \omega$. Then $f_{\alpha_{1}}<^{*} f_{\alpha}, f_{\alpha_{2}}<^{*} f_{\alpha}$ and $f_{\alpha}<^{*} g$. So there is $k \in \omega$ such that $\left\{\left\langle i, f_{\alpha}(i)\right\rangle: i\right\rangle$ $k\} \subseteq\left(H_{\alpha_{1}, \beta_{1}} \cap H_{\alpha_{2}, \beta_{2}}\right)$. Hence the intersection of any finite subcollection of $\mathcal{H}$ is finite and, by $(\mathrm{MA}+\neg \mathrm{CH})[5$, Corollary 8], there exists an infinite set $L \subseteq \omega \times \omega$ such that $L \backslash H_{\alpha, \beta}$ is finite for every $\alpha, \beta<\omega_{1}$.

Let $T_{i}=(\{i\} \times \omega) \subseteq \omega \times \omega$. It is clear that $T_{k} \cap H_{\alpha, \beta}$ is finite for every $\alpha, \beta<\omega_{1}$, $k \in \omega$. Thus $L \cap T_{k}$ is finite and we can find an infinite $\left\{n_{k}: k \in \omega\right\} \subseteq \omega$ and $\varphi \in{ }^{\omega} \omega$ such that $\left\{\left\langle n_{k}, \varphi\left(n_{k}\right)\right\rangle: k \in \omega\right\} \subseteq L$, so (i) holds.

EXAMPLE 3.4. (MA $+\neg C H)$ There exists a countable sequential space $X$ of weight $\omega_{1}$ with one non-isolated point which is not a quotient of any metric space of weight $\omega_{1}$.

Proof: Let $X=\{x\} \cup(\omega \times \omega)$. By (MA $+\neg \mathrm{CH})$, any subcollection of $\omega_{\omega} \omega$ having cardinality $\leqslant \omega_{1}$ has some majorant function from $\omega_{\omega} \omega[5$, Corollary 11]. So we can choose an increasing system $\left\{f_{\alpha}: \alpha<\omega_{1}\right\} \subseteq \omega_{\omega}$ such that for any $\langle i, j\rangle \in \omega \times \omega$ there is $\lambda<\omega_{1}$ with $j<f_{\lambda}(i)$.

We topologise $X$ as follows: points of $\omega \times \omega$ are isolated and the collection $\left\{B_{\alpha}: \alpha<\omega_{1}\right\}$ is a neighbourhood base at $x \in X$, where $B_{\alpha}=\{\langle i, j\rangle \in \omega \times \omega: j\rangle$ $\left.f_{\alpha}(i)\right\}$. The construction of $\left\{f_{\alpha}: \alpha<\omega_{1}\right\}$ implies $\{x\}=\bigcap\left\{B_{\alpha}: \alpha<\omega_{1}\right\}$ and hence the defined topology is regular $T_{1}$. Besides, since $\chi(X)<2^{\omega}$, the space $X$ is sequential [3, Theorem 1.1].

Let us assume that $X$ is a quotient of some metric space having weight $\omega_{1}$. By Theorem 2.3, $X$ has a sequential base $\mathcal{P}$ of cardinality $\omega_{1}$ which must consist of all points of $\omega \times \omega$ and some collection $\{P \subseteq X: x \in P\}$. Let $g \in \omega^{\omega} \omega$ be any majorant of $\left\{f_{\alpha}: \alpha<\omega_{1}\right\}$ which always exists as noted above. For every $P \in \mathcal{P}$ such that $x \in P$, we define $g_{P} \in{ }^{\omega} \omega$ as follows:

$$
g_{P}(i)= \begin{cases}\min \left\{j:\langle i, j\rangle \in\left(P \cap T_{i}\right)\right\} & \text { if }\left(P \cap T_{i}\right) \neq \emptyset \\ g(i) & \text { if }\left(P \cap T_{i}\right)=\emptyset\end{cases}
$$

where $i, j \in \omega, T_{i}=\{i\} \times \omega$. 
Pick the subcollection $\mathcal{P}^{*} \subseteq \mathcal{P}$ consisting of all points of $\boldsymbol{\omega} \times \boldsymbol{\omega}$ and the collection $\left\{P \in \mathcal{P}: x \in P, g_{P}\right.$ is a majorant of $\left.\left\{f_{\alpha}: \alpha<\omega_{1}\right\}\right\}$. We shall show that $\mathcal{P}^{*}$ is a sequential base of $X$. First note that for any $\left(P_{n}\right) \in \mathcal{P}_{z}$ (see Definition 2.1) there is $m \in \boldsymbol{\omega}$ such that $g_{P_{m}}$ is a majorant of $\left\{f_{\alpha}: \alpha<\omega_{1}\right\}$. If not, then for all $n \in \boldsymbol{\omega}$ there exists $\alpha(n)<\omega_{1}$ such that the condition $f_{\alpha(n)}<^{*} g_{P_{n}}$ is not satisfied. Choose $\alpha<\omega_{1}$ with $\alpha(n)<\alpha$ for all $n \in \omega$. Since $f_{\alpha(n)}<^{*} f_{\alpha}$ for every $n \in \omega$, the condition $f_{\alpha}<^{*} g_{P_{n}}$ is not true for all $n \in \omega$. Hence $P_{n} \subseteq B_{\alpha}$ does not hold for all $n \in \omega$ which is a conradiction. So there is $m \in \omega$ such that $g_{P_{m}}$ is a majorant of $\left\{f_{\alpha}: \alpha<\omega_{1}\right\}$. Since $P_{n+1} \subseteq P_{n}$ for all $n \in \omega, g_{P_{n}}$ is a majorant of $\left\{f_{\alpha}: \alpha<\omega_{1}\right\}$ for every $n>m$. So for any $\left(P_{n}\right) \in \mathcal{P}_{x}$ there is $m \in \omega$ with $P_{n} \in \mathcal{P}^{*}$ whenever $n>m$. Thus $\mathcal{P}^{*}$ is a sequential base of $X$ if for each $x \in X$ the collection $\mathcal{P}_{x}^{*} \subseteq\left(\mathcal{P}^{*}\right)^{\omega}$ is defined as follows:

$$
\mathcal{P}_{x}^{*}=\left\{\left(P_{n}^{*}\right) \in\left(\mathcal{P}^{*}\right)^{\omega}: \exists\left(P_{n}\right) \in \mathcal{P}_{x} \exists m \in \omega \forall n>m\left[P_{n}^{*}=P_{n}\right]\right\}
$$

Now apply Lemma 3.3 to the systems $\left\{f_{\alpha}: \alpha<\omega_{1}\right\},\left\{g_{P^{*}}: P^{*} \in \mathcal{P}^{*}\right\}$ to obtain $\varphi \in{ }^{\omega} \omega$ and $\left\{n_{k}: k \in \omega\right\} \subseteq \omega$ such that the condition (i) of Lemma 3.3 holds. Let $A=\left\{\left\langle n_{k}, \varphi\left(n_{k}\right)\right\rangle: k \in \omega\right\}$. Since for any $\alpha<\omega_{1}$ there is $k(\alpha) \in \omega$ with $f\left(n_{k}\right)<\varphi\left(n_{k}\right)$ for all $k>k(\alpha)$, we have that $x \in \bar{A} \backslash A$ and $A$ is not closed. On the other hand, for each $P^{*} \in \mathcal{P}^{*}$, we have $\varphi\left(n_{k}\right)<g_{P^{*}}\left(n_{k}\right)$ for all but finitely many $k \in \omega$. Therefore, if any $\left(P_{n}^{*}\right) \in \mathcal{P}_{x}^{*}$, then apply $\bigcap_{n} P_{n}^{*}=\{x\}$ to find $m \in \omega$ with $\varphi\left(n_{k}\right)<g P_{m}^{*}\left(n_{k}\right)$ for all $k \in \omega$. So $P_{m}^{*} \cap A=\emptyset$ and $A$ is closed. This contradiction shows that $X$ has no sequential base of cardinality $\omega_{1}$.

\section{A MAP CHARACTERISATION OF SOME SEQUENTIAL SPACES}

In this section we shall obtain some known classes of sequential spaces as particular quotient images of metric spaces.

THEOREM 4.1. The following are equivalent for a space $X$ and any cardinal $\tau$ :

(a) $X$ has a sequential base $\mathcal{P}$ such that $f n(\mathcal{P}) \leqslant \tau$.

(b) $X$ is an image of a metric space $M$ by a quotient map $f$ such that for every $x \in X$ the boundary of $f^{-1}(x)$ has cardinality $\leqslant \tau$.

Proof: (a) $\Rightarrow$ (b). Let $\mathcal{P}$ be a sequential base of $X$ and $M_{z_{1}\left(P_{n}\right)}$ be the set $X$ with the topology in which all points are open except for $x$ having $\left(P_{n}\right) \in \mathcal{P}_{x}$ as a neighbourhood base. We define the metric space $M$ to be the disjoint union of all spaces $M_{x,\left(P_{n}\right)}$, where $x \in X,\left(P_{n}\right) \in \mathcal{P}_{x}$. Then the natural map $f: M \rightarrow X$ (mapping a point into itself) is continuous, quotient and the boundary $\operatorname{Fr} f^{-1}(x)$ has cardinality $\leqslant \tau$ for every $x \in X$ (see [6, Theorem 2] where the case $\tau=\omega$ is considered).

(b) $\Rightarrow(a)$. This is proved just like $(\mathrm{a}) \Rightarrow(\mathrm{b})$ in Theorem 2.3 , but $f^{-1}(x)$ is replaced by $\operatorname{Fr} f^{-1}(x)$ for each $x \in X$. 
COROLlary 4.2. A space $X$ is $g$-first countable if and only if there exist a metric space $M$ and a quotient map $f: M \rightarrow X$ such that the boundary of $f^{-1}(x)$ is at most one point for each $x \in X$.

THEOREM 4.3. A space $X$ is symmetrisable [1] if and only if there exist a metric space $(M, \rho)$ and a quotient map $f: M \rightarrow X$ such that:

(i) the boundary of $f^{-1}(x)$ is at most one point $\xi(x)$ for each $x \in X$,

(ii) if a set $V$ is open in $X$ and $x \in V$, then there is $m \in \omega$ such that for every non-isolated point $x^{*} \in X \backslash V$, we have $\rho\left(\xi\left(x^{*}\right), f^{-1}(x)\right) \geqslant 1 / m$.

Proof: Let $d$ be a symmetric for $X$ and $P_{n}(x)=\left\{x^{*}: d\left(x^{*}, x\right)<1 / n\right\}$ for all $n \in \omega$. Then $\mathcal{P}=\left\{P_{n}(x): x \in X, n \in \omega\right\}$ is a sequential base of $X$ such that for each $x \in X$ the collection $\mathcal{P}_{x}$ consists of one element $\left(P_{n}(x)\right)$. Following the proof of $(\mathrm{a}) \Rightarrow$ (b) in Theorem 4.1, we shall consider the spaces $M_{x,\left(P_{n}(x)\right)}=M_{x}$, their disjoint union $M$ and the quotient map $f: M \rightarrow X$, so (i) holds. To prove (ii) note that the following metric $\rho$ is compatible with the topology of $M$ :

$$
\rho\left(x_{1}, x_{2}\right)= \begin{cases}1 / n & \text { if } x_{2} \in M_{x_{1}} \text { and } n=\min \left\{k: x_{2} \notin P_{k}\left(x_{1}\right)\right\} \\ \rho\left(x_{1}, x\right)+\rho\left(x, x_{2}\right) \text { if } x_{1}, x_{2} \in M_{x}, x_{1} \neq x_{2} \neq x \\ 1 \quad \text { in other cases, } x_{1} \neq x_{2} .\end{cases}
$$

Suppose $V \subseteq X$ is open, $x \in V, x^{*} \in X \backslash V$ and $\operatorname{Fr} f^{-1}\left(x^{*}\right)$ consists of one point $\xi\left(x^{*}\right)$. Then $x^{*} \notin P_{m}(x)$ and $x \notin P_{m}\left(x^{*}\right)$ for some $m \in \omega$. Since $\rho\left(x, x^{*}\right) \geqslant 1 / m$ whenever $x, x^{*} \in M_{x^{*}}$, we have that $\rho\left(\xi\left(x^{*}\right), f^{-1}(x)\right) \geqslant 1 / m$ for all $x, x^{*} \in X$.

Conversely, let $(M, \rho)$ and $f: M \rightarrow X$ satisfy the conditions in Theorem 4.3. For each $x \in X$ and $n \in \omega$, define

$$
P_{n}(x)= \begin{cases}\left\{x^{*}: \rho\left(\xi(x), f^{-1}\left(x^{*}\right)\right)<1 / n\right\} & \text { if } \operatorname{Fr} f^{-1}(x) \neq \emptyset \\ \{x\} & \text { if } \operatorname{Fr} f^{-1}(x)=\emptyset\end{cases}
$$

Trivially, $\mathcal{P}=\left\{P_{n}(x): x \in X, n \in \omega\right\}$ is a sequential base of $X$ if $\mathcal{P}_{x}$ consists of one element $\left(P_{n}(x)\right)$ for each $x \in X$. Define the symmetric $d: X \times X \rightarrow \mathbb{R}^{+}$as follows:

$$
d\left(x_{1}, x_{2}\right)= \begin{cases}1 / n & \text { if } n=\min \left\{k: x_{2} \notin P_{k}\left(x_{1}\right) \text { and } x_{1} \notin P_{k}\left(x_{2}\right)\right\} \\ 0 & \text { if } x_{1}=x_{2} .\end{cases}
$$

Now let $F$ be closed in $X$ and $x \in X \backslash F$. Pick $n \in \omega$ such that $P_{n}(x) \subseteq X \backslash F$. By (ii), find $m \in \omega$ such that $\rho\left(\xi\left(x^{*}\right), f^{-1}(x)\right) \geqslant 1 / m$ for all $x^{*} \in F$. If $k=\max \{m, n\}$ then $d(x, F) \geqslant 1 / k>0$. Further let $F$ be any subset of $X$ such that $d(x, F)>0$ for all $x \in X \backslash F$. Choose $n \in \omega$ with $P_{n}(x) \cap F \neq \emptyset$, so $F$ is closed. Thus $d$ is a symmetric on $X$ which is compatible with the topology. 
REMARK. Another map characterisation of symmetrisable spaces has been obtained in [4, Theorem 11].

The following notion was introduced in [8]. We shall find it convenient to formulate it as follows:

Definition 4.4: A space $X$ is $g$-second countable [8] if $X$ has a countable sequential base with finite fan-number.

THEOREM 4.5. A space $X$ is $g$-second countable if and only if there exist a separable metric space $M$ and a map $f: M \rightarrow X$ such that for every $x \in X$, a point $\xi(x) \in f^{-1}(x)$ may be found with the following property:

(i) if a set $G$ is open in $M$ and for every $x \in f(G)$ we have that $\xi(x) \in G$, then the set $f(G)$ is open in $X$.

Proof: The proof of "if" part is quite similar to the proof of $(a) \Rightarrow(b)$ in Theorem 2.3 , but instead of $f^{-1}(x)$ we take $\xi(x)$. The "only if" is essentially the same as the proof of $(\mathrm{b}) \Rightarrow(\mathrm{a})$ in Theorem 2.3, but for each $x \in X$, the collection $\mathcal{P}_{x}$ consists of only one element denoted by $\xi(x)$.

It is proved in [2, Theorem 6.1] that a space $X$ has a point-countable sequential base if and only if $X$ is a quotient $s$-image of a metric space. We conclude this paper with a result which is proved just like Theorem 2.7, and its proof is thus omitted.

THEOREM 4.6. The following are equivalent for a space $X$ and any cardinal $\tau$ :

(a) $X$ is the image of a metric space by a quotient map with countable fibers.

(b) $X$ has a point-countable sequential base $\mathcal{P}$ with countable fan-number.

\section{REFERENCES}

[1] A.V. Arhangel'skii, 'Mappings and spaces', Russian Math. Surveys 21 (1961), 115-162.

[2] G. Gruenhage, E. Michael and Y. Tanaka, 'Spaces determined by point-countable covers', Pacif. J. Math. 113 (1984), 303-331.

[3] V.I. Malyhin and B.E. Šapirovskiǐ, 'Martin's axiom and properties of topological spaces', Soviet Math. Dokl. 14 (1973), 1746-1751.

[4] S. Nedev, 'O-metrizable spaces', Trans. Moscow Math. Soc. 24 (1971), 201-236.

[5] M.E. Rudin, 'Martin's axiom', in Handbook of mathematical logic (NorthHolland Publishing Company, Amsterdam, 1977), pp. 491-502.

[6] R. Sirois-Dumais, 'Quasi- and weakly-quasi-first-countable spaces', Topology Appl. 11 (1980), 223-230.

[7] R. Sirois-Dumais and S. Willard, 'Quotient-universal sequential spaces', Pacif. J. Math. 66 (1976), 281-289.

[8] F. Siwiec, 'On defining a space by a weak base', Pacif. J. Math. 52 (1974), 223-244.

Department of Mathematics 